\title{
RNA-seq analysis identifies key long non-coding RNAs connected to the pathogenesis of alcohol-associated head and neck squamous cell carcinoma
}

\author{
VICKY YU $^{1 *}$, PRANAV SINGH ${ }^{1 *}$, ELHAM RAHIMY ${ }^{1}$, HAO ZHENG $^{1}$, SELENA Z. KUO ${ }^{1}$, \\ ELIZABETH KIM $^{1}$, JESSICA WANG-RODRIGUEZ ${ }^{2}$ and WEG M. ONGKEKO ${ }^{1}$
}

${ }^{1}$ Department of Surgery, Division of Otolaryngology-Head and Neck Surgery; ${ }^{2}$ Department of Pathology, Veterans Administration Medical Center, University of California San Diego, San Diego, CA 92161, USA

Received February 22, 2015; Accepted March 24, 2016

DOI: $10.3892 / \mathrm{ol} .2016 .4972$

\begin{abstract}
Alcohol consumption has been implicated in the pathogenesis of head and neck squamous cell carcinoma (HNSCC), although its mechanism is poorly understood. Recent advances in the identification and understanding of long non-coding RNAs (IncRNAs) have indicated that these molecules have a profound effect on numerous biological processes, including tumorigenesis and oncogenesis. The present authors hypothesize that alcohol-mediated dysregulation of lncRNAs is a key event in HNSCC pathogenesis. An in silico differential expression analysis utilizing RNA sequencing (RNA-seq) data from 34 HNSCC patients, which included alcohol drinkers and non-alcohol drinkers, identified a panel of lncRNAs that were dysregulated due to alcohol consumption. Normal oral keratinocytes were then exposed to ethanol and acetaldehyde to validate the RNA-seq results. Two lncRNAs that were differentially expressed due to alcohol consumption were identified from RNA-seq analysis of the clinical data: $\operatorname{lnc}-P S D 4-1$ and lnc-NETO-1. Oral keratinocytes exposed to alcohol and acetaldehyde demonstrated dysregulation of these two lncRNAs, thus validating the results of RNA-seq analysis. In addition, low expression of the $\operatorname{lnc}-P S D 4-1$ isoform, $\operatorname{lnc}$-PSD4-1:14, exhibited a strong correlation with high survival rates in a Cox proportional hazards regression model. Therefore, these IncRNAs may play a key role in the early pathogenesis of HNSCC, since they are dysregulated in both clinical data and in vitro experiments mimicking the effects of alcohol use.
\end{abstract}

Correspondence to: Dr Weg M. Ongkeko, Department of Surgery, Division of Otolaryngology-Head and Neck Surgery, University of California San Diego, 9500 Gillman Drive, La Jolla, San Diego, CA 92161, USA

E-mail: rongkeko@ucsd.edu

${ }^{*}$ Contributed equally

Key words: alcohol, acetaldehyde, RNA-seq, long non-coding RNAs, head and neck squamous cell carcinoma, epigenetics

\section{Introduction}

Alcohol and tobacco use are associated with $\geq 75 \%$ of all head and neck squamous cell carcinomas (HNSCCs) (1). In addition, alcohol has been reported to independently increase the risk of cancer (2). Despite compelling evidence suggests that alcohol plays a key role in the pathogenesis of HNSCC, its molecular mechanism remains poorly understood (2). Alcohol is speculated to increase the risk of cancer by impairing DNA-repair genes or folate metabolism (2). However, previous studies have suggested that it is not alcohol, but instead its metabolite acetaldehyde, the major contributor to HNSCC progression $(2,3)$. Acetaldehyde is produced by alcohol dehydrogenases in the intestine, kidney, liver and oral cavity, and has been proposed to exert mutagenic effects, including DNA cross-linking, chromatid exchange, aneuploidy and other chromosomal abnormalities (3). The present study specifically focuses on the effect of alcohol exposure on HNSCC, and proposes that ethanol or its derivative acetaldehyde alters the expression of key long non-coding RNAs (IncRNAs) that may be critical in the pathogenesis of HNSCC.

The human genome sequence is composed primarily of non-coding RNAs (ncRNAs), with only $2 \%$ of RNAs coding for proteins $(4,5)$. Previously, ncRNAs were considered to be transcriptional noise (4). However, it has been recently reported that ncRNAs are important in transcriptional and post-transcriptional processes (4). Recent studies have revealed that ncRNAs influence messenger RNA (mRNA) translation and chromatin modifications (5). Since previous studies have demonstrated that alcohol is able to regulate ncRNAs $(6,7)$, it is possible that the role of ncRNAs as epigenetic regulators could account for the effects of alcohol in the pathogenesis of HNSCC.

The present study focused on IncRNAs, which are ncRNAs of $>200$ nucleotides in length. lncRNAs have been reported to play a critical role in cancer progression through the modification of transcription factors associated with regulation of oncogenes, tumor suppressor proteins, self-renewal and differentiation $(8,9)$. lncRNAs regulate transcription factors by acting as chromatin modifiers and direct transcriptional regulators $(8,10,11)$. This regulation has been demonstrated to occur either in cis (in close proximity to the transcribed lncRNA) or in trans (far from 
the transcription site) (12). Therefore, alcohol-dysregulated lncRNAs could play critical roles in the inhibition of tumor suppressors or the activation of oncogenes that are required for the malignant transformation of normal oral epithelial cells.

Using RNA sequencing (RNA-seq) technology, the present study identified a panel of lncRNAs that were differentially expressed between alcohol drinkers and non-alcohol drinkers among HNSCC patients. This panel was partially validated in vitro in normal oral keratinocytes treated with clinically relevant levels of ethanol and acetaldehyde.

\section{Materials and methods}

RNA-seq analysis. An in silico differential expression analysis was conducted utilizing publicly available RNA-seq libraries obtained from The Cancer Genome Atlas (TCGA; https://tcga-data.nci.nih.gov/tcga), which comprised 34 HNSCC patients, 17 of which were alcohol drinkers and 17 non-alcohol drinkers. Within each category of alcohol drinkers and non-alcohol drinkers, 12 patients were tobacco smokers and 5 were non-tobacco smokers (Table I). The libraries were generated by TCGA utilizing a Genome Analyzer IIx (Illumina Inc., San Diego, CA, USA), which resulted in paired-end RNA-seq libraries with insert sizes of 200 bp-5 kb. These RNA-seq files were next position-sorted, indexed and aligned to a human reference genome (hg19). The files were then annotated with a browser extensible data file containing 32,108 human lncRNA transcripts, which was downloaded from LNCipedia (http://www.lncipedia.org/) (13). The bedtools (http://bedtools.readthedocs.io/en/latest/) (14) utility coverageBed was then used to generate lncRNA read counts (integer values of expression levels) by calculating the number of alignments from each RNA-seq file that overlapped with each individual lncRNA provided by the annotation file from LNCipedia. A total of 13,338 lncRNAs displayed reads generated in the RNA-seq libraries.

These read counts were then utilized for IncRNA differential expression analysis, which compared alcohol drinkers vs. non-alcohol drinkers using the R/Bioconductor software package edgeR (version 3.4.2; http://www.bioconductor. org/packages). The read counts were normalized within edgeR based on the relative library sizes of each cohort. The differential expression analysis implemented in edgeR utilized an empirical Bayes estimation and exact tests based on the negative binomial distribution of the reads (15). From this comparison, a list of differentially expressed lncRNAs with false discovery rates $<5 \%$ was compiled in HNSCC patients who were alcohol drinkers vs. those who were non-alcohol drinkers (Table II).

Cell culture. In vitro experiments were performed on OKF4 and OKF6, two noncancerous cell lines obtained from the laboratory of Dr James Rheinwald at Harvard Medical School (Harvard University, Boston, MA, USA). Normal oral keratinocytes from the floor of the mouth were used, since the hypothesis proposed by the present authors concerns the initial steps in the pathogenesis of alcohol-induced oropharyngeal cancer.

The oral keratinocytes were cultured in $1 \mathrm{X}$ Keratinocyte-serum-free medium (SFM) with L-glutamine (catalogue no. 17005-042), supplemented with $0.2 \mathrm{ng} / \mathrm{ml}$ human recombinant epidermal growth factor (EGF) type B (amino acids 1-53), $25 \mu \mathrm{g} / \mathrm{ml}$ bovine pituitary extract (BPE), $0.3 \mathrm{mM}$ calcium chloride, $100 \mathrm{U} / \mathrm{ml}$ penicillin and $100 \mu \mathrm{g} / \mathrm{ml}$ streptomycin (all from Gibco; Thermo Fisher Scientific, Inc., Waltham, MA, USA), at $37^{\circ} \mathrm{C}$ and $5 \% \mathrm{CO}_{2}$. Upon reaching $30 \%$ confluency, OKF4 and OKF6 cells were cultured with equal parts of supplemented Keratinocyte-SFM and DFK medium, which was prepared with equal parts of Dulbecco's modified Eagle's medium (catalogue no. 21068-028; Gibco; Thermo Fisher Scientific, Inc.) and Ham's F-12 nutrient mixture (catalogue no. 11765-054; Thermo Fisher Scientific, Inc.), and supplemented with $0.2 \mathrm{ng} / \mathrm{ml}$ EGF type B (amino acids 1-53), $25 \mu \mathrm{g} / \mathrm{ml} \mathrm{BPE}, 2 \mathrm{mM}$ L-glutamine, $100 \mathrm{U} / \mathrm{ml}$ penicillin and $100 \mu \mathrm{g} / \mathrm{ml}$ streptomycin.

Ethanol/acetaldehyde treatments. Two independent experiments regarding cell treatments were conducted in the present study, one for ethanol and one for acetaldehyde. For both, two biological replicates were performed. For the alcohol experiments, the two cell lines were treated with increasing dosages of 200 proof ethanol $(0,20,50$ and $170 \mu \mathrm{M})$. The concentrations of ethanol were selected based on their toxicity, and an 3-(4,5-dimethylthiazol-2-yl)-2,5-diphenyltetrazolium bromide (MTT) assay was performed to determine the levels of cell proliferation at varying ethanol concentrations (Fig. 1). To represent long-term alcohol use, cells were treated every $24 \mathrm{~h}$ with ethanol diluted in medium (equal parts Keratinocyte-SFM and DFK) for a period of 28 days. The ethanol and the medium were replaced daily. The ethanol-treated culture plates were covered with plastic paraffin film while incubating at $37^{\circ} \mathrm{C}$ to minimize ethanol evaporation.

The above treatment was repeated with $17.82 \mathrm{M}$ acetaldehyde (catalogue no. SHBD3908V; Sigma-Aldrich, St. Louis, MO, USA), since this is the first metabolite of ingested alcohol in the human body (3). OKF4 and OKF6 cells were treated with acetaldehyde at concentrations of $0,75,150,300$ and $1,000 \mu \mathrm{M}$ for $48 \mathrm{~h}$, with acetaldehyde added every $4 \mathrm{~h}$ and the medium (equal parts Keratinocyte-SFM and DFK) replaced every $8 \mathrm{~h}$. The appropriate concentrations of acetaldehyde were determined from previous studies (16). Due to the volatility, toxicity and short half-life of acetaldehyde, cells could not be treated for 28 days (as they had been for ethanol). The plates were also covered with plastic paraffin film to minimize evaporation of acetaldehyde while incubated at $37^{\circ} \mathrm{C}$. Cell lines were passaged at $30-80 \%$ confluency prior to harvesting.

Reverse transcription-quantitative polymerase chain reaction (RT-qPCR). Upon completion of alcohol and acetaldehyde treatments, cells were harvested, and total cell lysates were collected. RNA was extracted using SurePrep RNA Isolation kit (Thermo Fisher Scientific, Inc.). Complementary DNA was synthesized according to the manufacturer's protocol, using LncProfiler qPCR Array kit (catalogue no. RA900A-1; System Biosciences, Mountain View, CA, USA). qPCR was performed using SYBR Green reagent (Applied Biosystems; Thermo Fisher Scientific, Inc.) in a StepOnePlus Real-time PCR System (Applied Biosystems; Thermo Fisher Scientific, Inc.) for $2 \mathrm{~min}$ at $50^{\circ} \mathrm{C}, 95^{\circ} \mathrm{C}$ for $10 \mathrm{~min}$, and 40 cycles of $95^{\circ} \mathrm{C}$ for $15 \mathrm{sec}$ and $60^{\circ} \mathrm{C}$ for $1 \mathrm{~min}$. Gene expression levels with gene-specific primers (Eurofins MWG Operon, Louisville, 
Table I. Demographic characteristics of 34 patients with head and neck squamous cell carcinoma included in the present in silico analysis, with categorical breakdowns of drinking status, smoking status, vital state, gender, tumor site, stage and grade.

\begin{tabular}{|c|c|c|c|c|c|}
\hline Variables & $\begin{array}{c}\text { Total } \\
\text { patients } \\
(\%) \mathrm{n}=34\end{array}$ & $\begin{array}{c}\text { Alcohol drinkers } \\
\text { and tobacco smokers } \\
\qquad \%) \mathrm{n}=12\end{array}$ & $\begin{array}{l}\text { Alcohol drinkers but } \\
\text { non-tobacco smokers } \\
\qquad \%) n=5\end{array}$ & $\begin{array}{l}\text { Non-alcohol drinkers } \\
\text { but tobacco smokers } \\
\qquad(\%) \mathrm{n}=12\end{array}$ & $\begin{array}{c}\text { Non-alcohol drinkers } \\
\text { or tobacco smokers } \\
(\%) \mathrm{n}=5\end{array}$ \\
\hline \multicolumn{6}{|l|}{ Gender } \\
\hline Male & $25(74)$ & $10(83)$ & $3(60)$ & $8(67)$ & $4(80)$ \\
\hline Female & $9(26)$ & $2(17)$ & $2(40)$ & $4(33)$ & $1(20)$ \\
\hline \multicolumn{6}{|l|}{ Drinks per day } \\
\hline None & $17(50)$ & $0(0)$ & $0(0)$ & $12(100)$ & $5(100)$ \\
\hline $0-2$ & $14(41)$ & $11(92)$ & $3(60)$ & $0(0)$ & $0(0)$ \\
\hline$>2$ & $3(9)$ & $1(8)$ & $2(40)$ & $0(0)$ & $0(0)$ \\
\hline \multicolumn{6}{|l|}{ Vital state } \\
\hline Deceased & $11(32)$ & $4(33)$ & $0(0)$ & $6(50)$ & $1(20)$ \\
\hline Alive & $23(68)$ & $8(67)$ & $5(100)$ & $6(50)$ & $4(80)$ \\
\hline \multicolumn{6}{|l|}{ Tumor site } \\
\hline Oral & $24(70)$ & $8(67)$ & $3(60)$ & $9(75)$ & $4(80)$ \\
\hline Pharyngeal & $4(12)$ & $1(8)$ & $2(40)$ & $0(0)$ & $1(20)$ \\
\hline Laryngeal & $6(18)$ & $3(25)$ & $0(0)$ & $3(25)$ & $0(0)$ \\
\hline \multicolumn{6}{|l|}{ Stage } \\
\hline Low (I, II) & $5(15)$ & $2(17)$ & $2(40)$ & $1(8)$ & $0(0)$ \\
\hline High (III, IV) & $29(85)$ & $10(83)$ & $3(60)$ & $11(92)$ & $5(100)$ \\
\hline \multicolumn{6}{|l|}{ Grade } \\
\hline GX & $1(3)$ & $0(0)$ & $1(20)$ & $0(0)$ & $0(0)$ \\
\hline G1-G2 & $26(76)$ & $7(58)$ & $4(80)$ & $10(83)$ & $5(100)$ \\
\hline G3-G4 & $7(21)$ & $5(42)$ & $0(0)$ & $2(17)$ & $0(0)$ \\
\hline
\end{tabular}
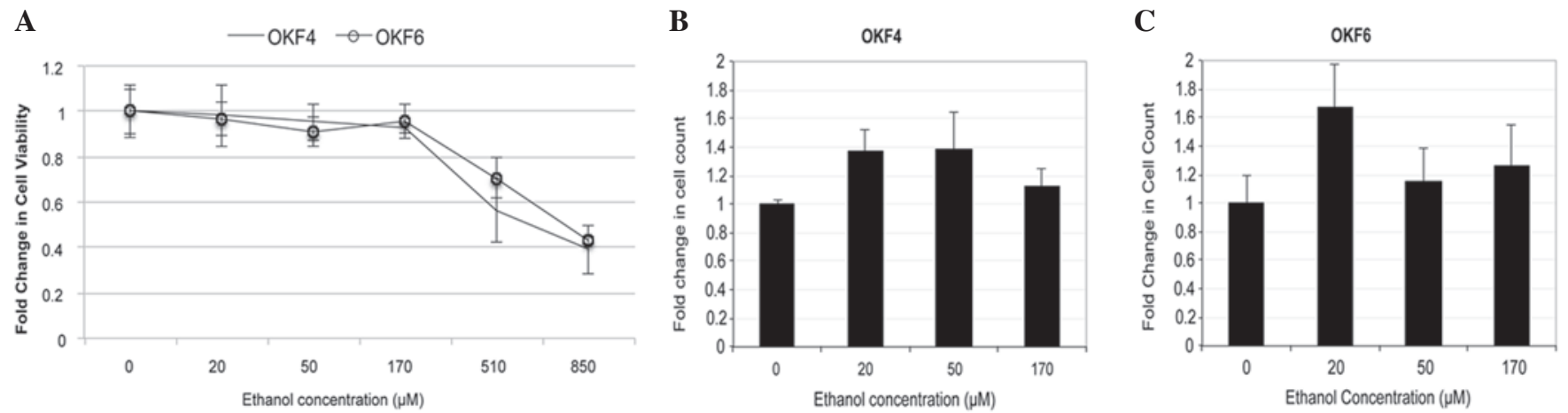

Figure 1. (A) Cell proliferation and viability in alcohol-treated cells. Cell viability with increasing levels of ethanol concentration was measured in (B) OKF4 and (C) OKF6 cell lines by 3-(4,5-dimethylthiazol-2-yl)-2,5-diphenyltetrazolium bromide assay. Error bars denote standard deviation.

KY, USA; Table II) and error bars were calculated utilizing the $2^{-\Delta \Delta \mathrm{Cq}}$ method (17), with non-treated cells acting as a control for the ethanol and acetaldehyde treatments, and $g l y c$ eraldehyde 3-phosphate dehydrogenase serving as a control for endogenous gene expression.

Survival data analysis. Utilizing the original 34 patient cohort and clinical information provided by the TCGA, the expression levels of key dysregulated lncRNAs were correlated with the patients' long-term survival. The expression levels of the IncRNAs were classified as either increased or decreased depending on whether they fell above or below the median value. A Cox proportional hazards regression model was then applied to determine both univariate and multivariate survival hazard ratios (HRs) for the lncRNAs, based on a decreased expression (Table III). A Kaplan-Meier survival curve was used to illustrate the correlation between lncRNA expression and survival.

\section{Results and Discussion}

The purpose of the present study was to identify key lncRNAs implicated in the pathogenesis of HNSCC, since lncRNAs are 


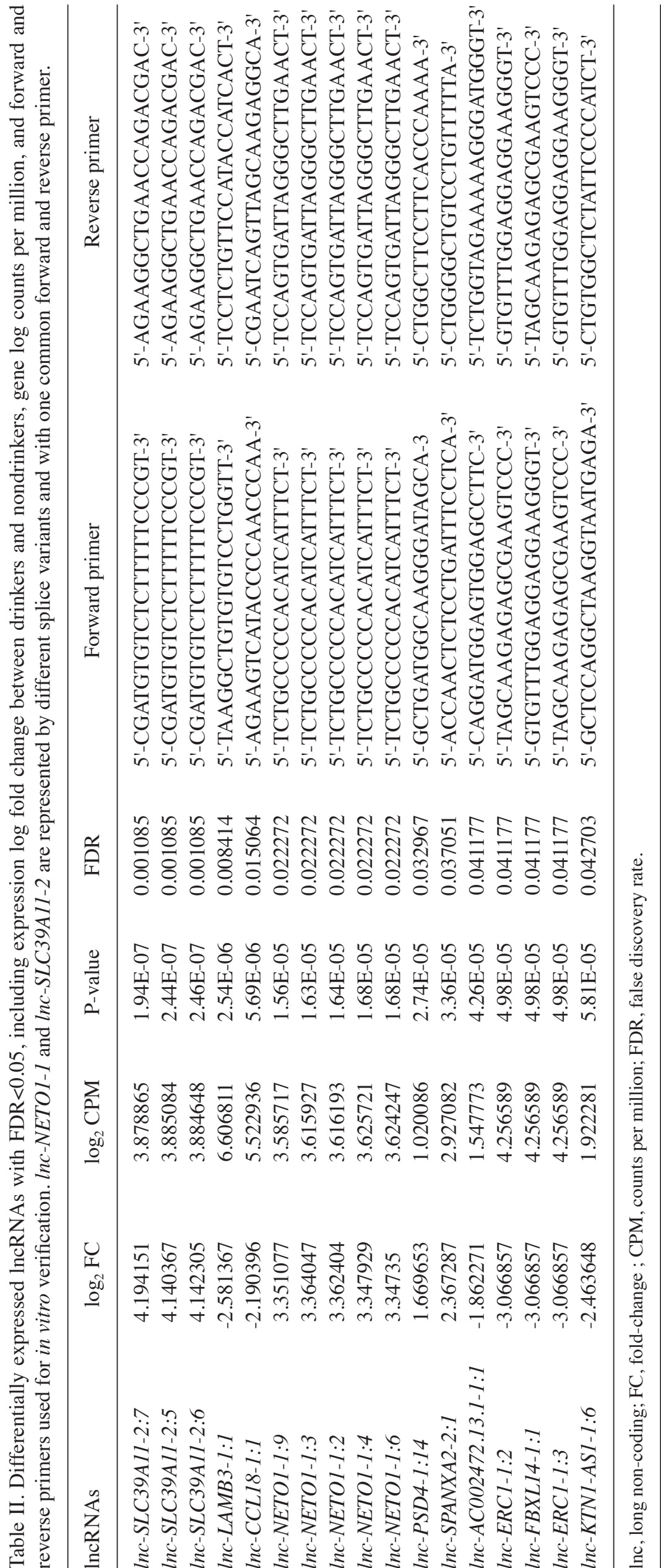


known to regulate transcription factors associated with regulation of oncogenes, tumor suppressor proteins, self-renewal and differentiation $(8,9)$. Recent studies have demonstrated that dysregulated lncRNA expression could mark the progression of a disease (18). IncRNAs may also serve as an indicator of patient survival independent of other variables (19). In addition, IncRNAs have previously been implicated in HNSCC and other types of epithelial cancer (20). Therefore, lncRNAs may aid the understanding of the molecular basis of HNSCC, thus enabling advances in early detection and identification of novel therapeutic targets aimed to improve patient prognosis.

Determination of ethanol concentrations. MTT assays were performed in the present study to determine the ethanol concentrations required for cell treatments and to evaluate cell proliferation prior and subsequent to treatment. Concentrations of $0,20,50$ and $170 \mu \mathrm{M}$ ethanol were selected for the experimental assays (Fig. 1). However in cell culture, $170 \mu \mathrm{M}$ ethanol exhibited high toxicity against normal oral keratinocytes, and therefore was not used in subsequent experiments.

Identification of alcohol-dysregulated IncRNAs from HNSCC patient samples. The expression patterns of 32,108 lncRNAs were examined in the present study, 13,338 of which were detected within the present cohort of $34 \mathrm{HNSCC}$ patients. Of those 13,338 lncRNAs, 11 were differentially expressed between alcohol drinkers and non-alcohol drinkers, with several lncRNAs represented by multiple isoforms (Table II). Of the identified lncRNAs, 4 were upregulated and 7 were downregulated, with fold-changes ranging from $\geq 3.5$ to 18.5 and false discovery rates $<5 \%$. The small panel of 1 ncRNAs differentially expressed between alcohol drinkers and non-alcohol drinkers suggests that the mechanism by which alcohol contributes to the pathogenesis of HNSCC involves certain lncRNAs.

In vitro validation of IncRNAs differentially expressed in clinical samples. The lncRNAs identified by RNA-seq analysis in the present study were evaluated in vitro by measuring their relative expression levels in two normal keratinocyte cell lines, which were exposed to ethanol concentrations of $0,20,50$ and $170 \mu \mathrm{M}$ (Fig. 3) and acetaldehyde concentrations of $0,75,150$, 300 and $1,000 \mu \mathrm{M}$ (Fig. 4), via RT-qPCR. Of the 11 lncRNAs identified in the RNA-seq analysis, two were verified in vitro: lnc-PSD4-1 (including the isoform, lnc-PSD4-1:14) and lnc-NETO1-1, whose expression levels were increased in the treated samples, compared with the non-treated controls. These results suggest that the above lncRNAs may be important in the pathogenesis and progression of alcohol-associated HNSCC.

For the ethanol-treated OKF4 cell line, both PSD4-1 and NETO1-1 exhibited negligible changes in their expression levels when $20 \mu \mathrm{M}$ ethanol was used, while a 2 -fold increase in their expression levels was observed in $50 \mu \mathrm{M}$ ethanol-treated cells. For OKF6 cells, $20 \mu \mathrm{M}$ ethanol exposure resulted in a 5-fold increase in the expression levels of PSD4-1 and NETO1-1, while $50 \mu \mathrm{M}$ ethanol exposure resulted in a 2-fold increase in their expression levels, compared with the control. The higher expression levels observed in the $20 \mu \mathrm{M}$ ethanol-treated cells vs. the $50 \mu \mathrm{M}$ ethanol-treated cells may

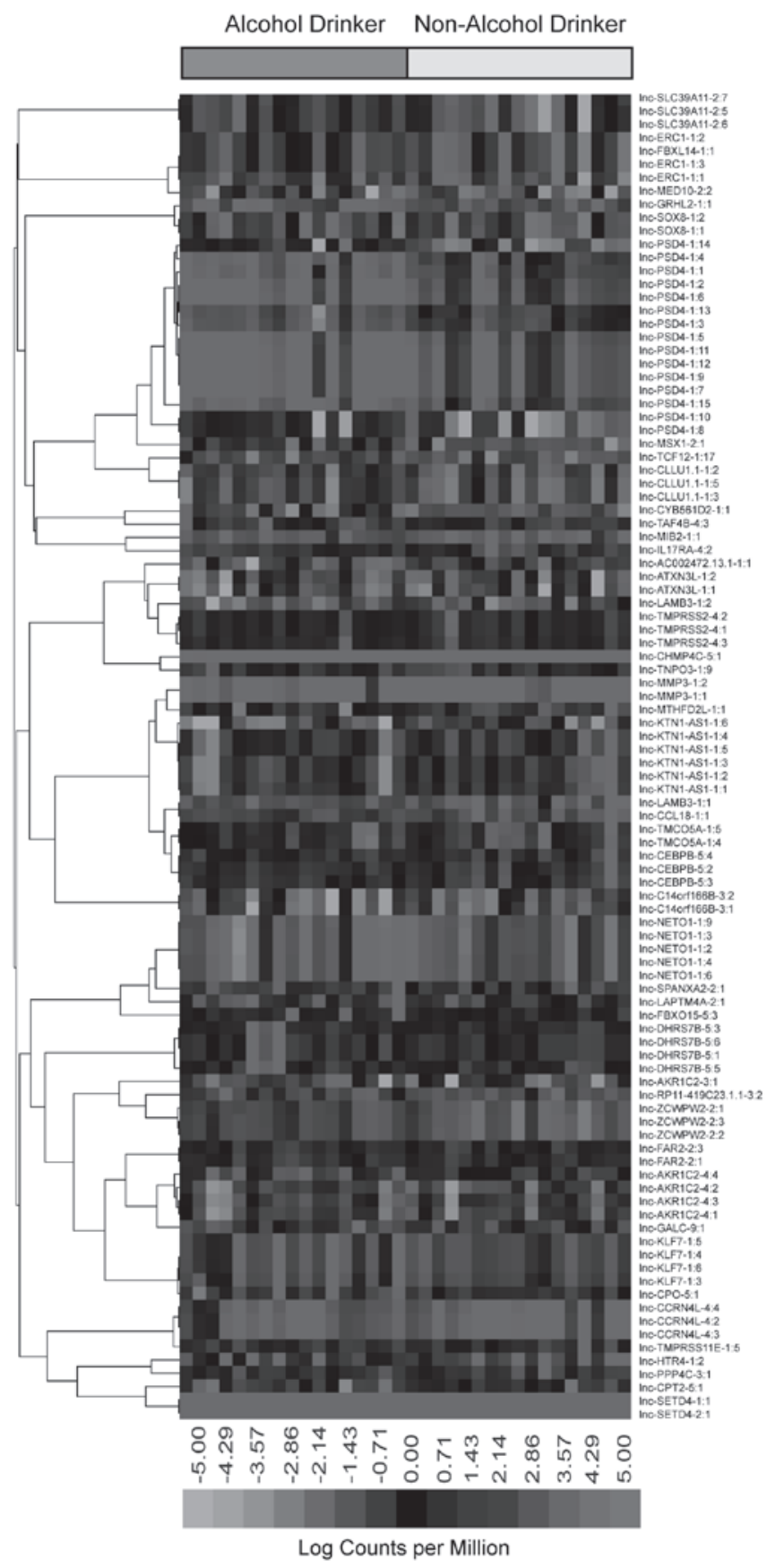

Figure 2. Heatmap of alcohol-dysregulated lncRNAs. Heatmap depicting normalized lncRNA expression levels (in the form of counts per million) across the alcohol drinker and non-alcohol drinker cohorts in 34 patients with head and neck squamous cell carcinoma. The top 100 differentially expressed lncRNAs are presented, including those whose false discovery rate was not $<0.05$. lnc, long non-coding.

be due to the $50 \mu \mathrm{M}$ ethanol concentration being too toxic for OKF6 cells, in contrast to the OKF4 cell line, where $50 \mu \mathrm{M}$ ethanol demonstrated the largest effect.

While the ethanol treatment used in the present study mimics the physiological levels of alcohol in the body, ethanol is less carcinogenic than its metabolic derivative acetaldehyde (3). Therefore, acetaldehyde treatment may be a more accurate in vitro model of the role of alcohol in HNSCC than ethanol treatment. In general, in acetaldehyde-treated samples, both PSD4-1 and NETO1-1 displayed higher expression levels, 
Table III. Cox proportional hazards regression model for the lncRNA PSD4-1:14. Survival information, including HR and P-value, for $\operatorname{lnc}-P S D 4-1: 14$ in both univariate and multivariate models demonstrates a strong correlation between low expression of PSD4-1:14 and improved overall survival.

\begin{tabular}{lcccr}
\hline Low expression & $\begin{array}{c}\text { Univariate HR } \\
(95 \% \mathrm{CI})\end{array}$ & P-value & $\begin{array}{c}\text { Multivariate HR } \\
(95 \% \text { CI })\end{array}$ & P-value \\
\hline lnc-PSD4-1:14 & 0.267150 & 0.047926 & 0.236208 & 0.034013 \\
& $(0.072234-0.988021)$ & & $(0.062212-0.896836)$ & \\
\hline
\end{tabular}

lnc, long non-coding; HR, hazard ratio; CI, confidence interval.

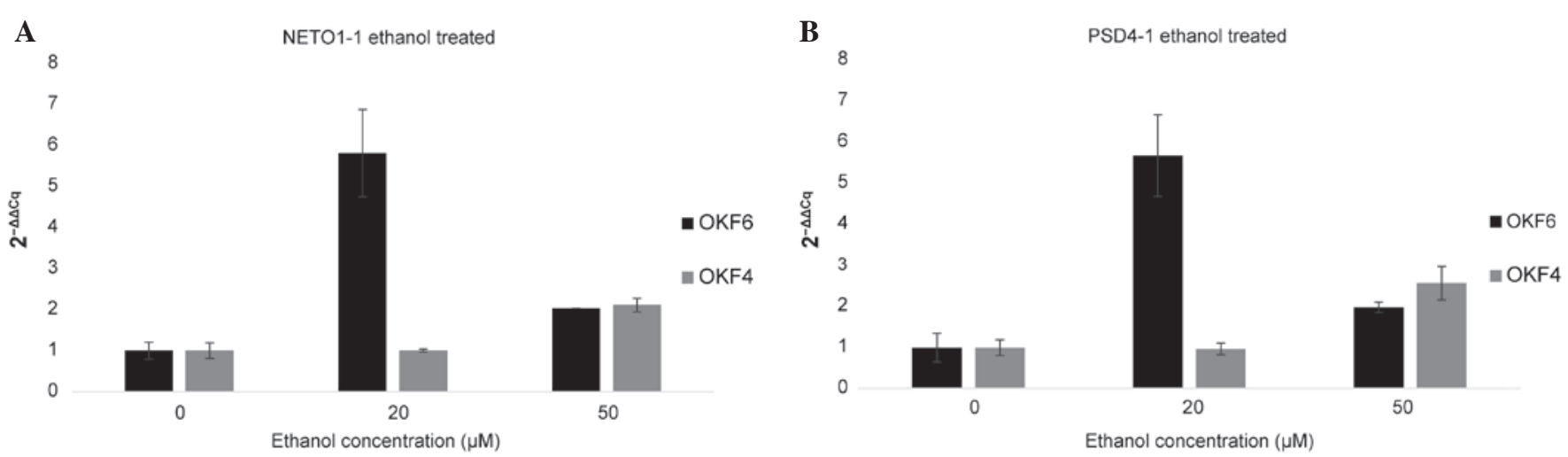

Figure 3. Validation of alcohol-dysregulated lncRNAs identified in vitro with ethanol-treated cell lines. Reverse transcription-quantitative polymerase chain reaction analysis of ethanol-treated OKF4 and OKF6 cell lines demonstrated that (A) lnc-NETO1-1 and (B) lnc-PSD4-1 are dysregulated by alcohol. Error bars represent the standard deviation, as calculated by the $2^{-\Delta \Delta C q}$ method. lnc, long non-coding.

A

NETO1-1 acetaldehyde treated samples
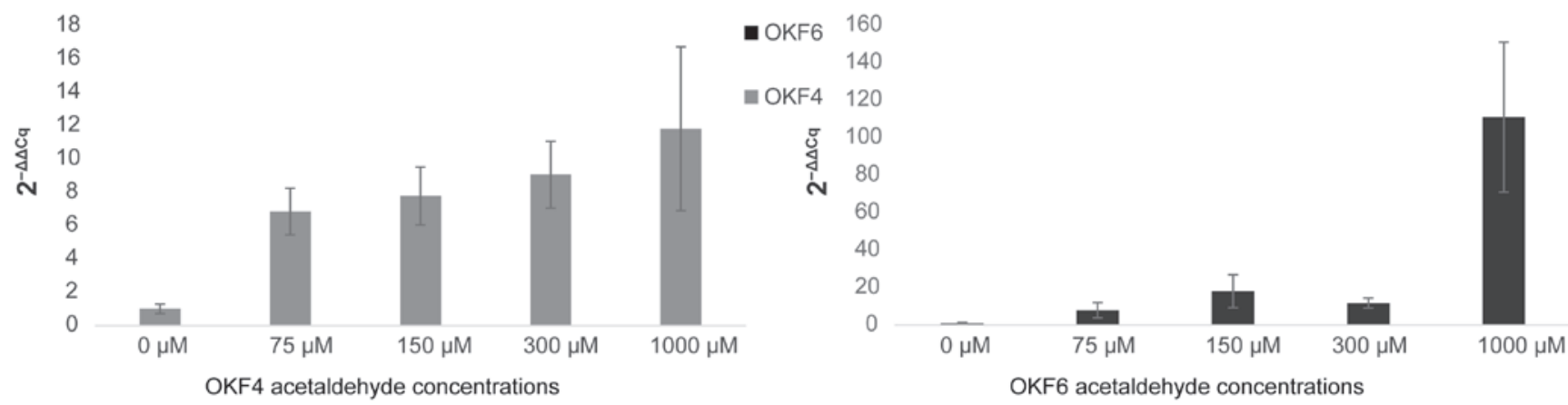

B

PSD4-1 acetaldehyde treated samples
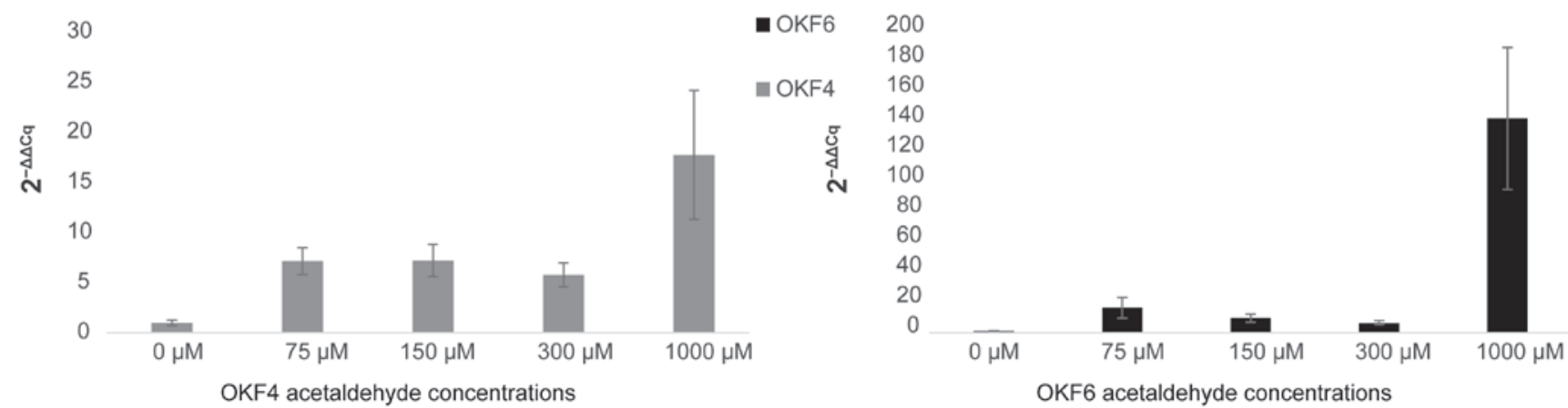

Figure 4. Validation of alcohol-dysregulated lncRNAs in vitro in acetaldehyde-treated cell lines. Reverse transcription-quantitative polymerase chain reaction analysis of acetaldehyde-treated OKF4 and OKF6 cell lines demonstrated that (A) lnc-NETO1-1 and (B) lnc-PSD4-1 are dysregulated by acetaldehyde. Error bars represent the standard deviation, as calculated by the $2^{-\Delta \Delta C q}$ method. lnc, long non-coding. 


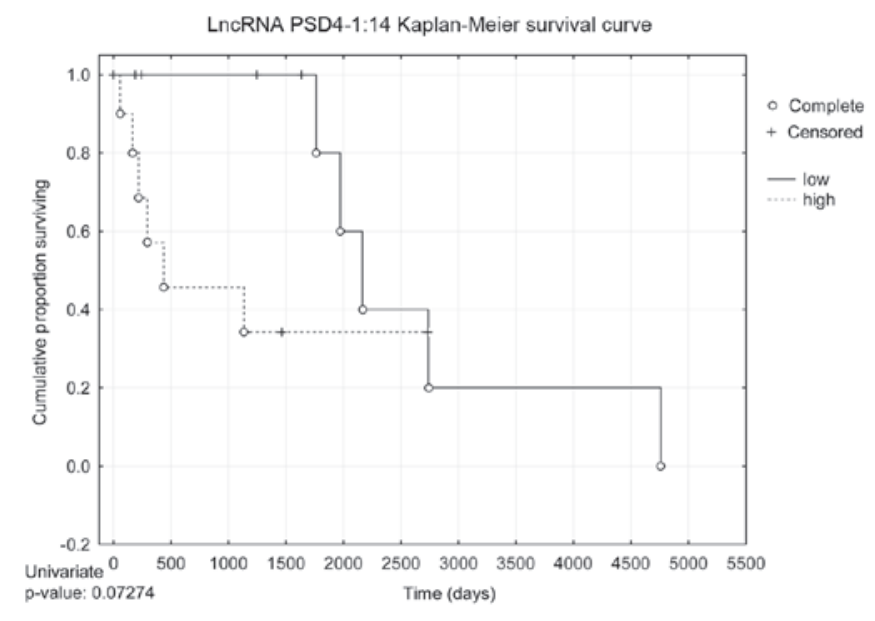

Figure 5. Kaplan-Meier survival curve for $\operatorname{lnc}$-PSD4-1:14. Kaplan-Meier survival graph depicted a correlation between low expression levels of lnc-PSD4-1:14 and high survival in patients with head and neck squamous cell carcinoma. lnc, long non-coding.

correlating with higher concentrations of acetaldehyde. In OKF4 cells treated with $1,000 \mu \mathrm{M}$ acetaldehyde, NETO1-1 and PSD4-1 demonstrated a 12-fold and 16-fold increase in expression, respectively, compared with the control. In OKF6 cells treated with the same concentration of acetaldehyde, both PSD4-1 and NETO1-1 demonstrated a >100-fold increase in expression. In the present study, $1,000 \mu \mathrm{M}$ was selected as the upper range of acetaldehyde concentration, since the treatment of the OKF4 and OKF6 cell lines was limited to only $48 \mathrm{~h}$ due to the acetaldehyde's volatility. This concentration is considered to be more representative of the actual exposure to alcohol experienced by patients with a long history of alcohol use.

Survival data. In addition to the aforementioned in vitro tests, long-term survival analysis correlating NETO1-1 and PSD4-1:14 expression levels and patient outcomes was conducted. NETO1-1 did not exhibit any significant correlation with patient survival. By contrast, low expression levels of PSD4-1:14 were highly correlated with overall better patient survival in both univariate $(\mathrm{HR}, 0.267150 ; \mathrm{P}=0.047926)$ and multivariate (HR, 0.236208; $\mathrm{P}=0.034013$ ) Cox proportional hazards regression models (Table III). The univariate Kaplan-Meier survival curve in Fig. 5 demonstrates that low expression levels of PSD4-1:14 correlate with better patient survival. Although the Kaplan-Meier survival curve is not statistically significant, it approached $\sim \mathrm{P}=0.05$, and would likely be statistically significant in a larger sample size.

In summary, the present findings have demonstrated an association between alcohol-associated HNSCC and increased expression of the 1ncRNAs NETO1-1 and PSD4-1:14. The increased expression of NETO1-1 and PSD4-1:14 in both HNSCC patient clinical samples and in vitro models of alcohol usage suggest that these lncRNAs may act as activators of oncogenes. Previous studies have demonstrated that lncRNAs in an antisense orientation are able to control the transcription of mRNAs and oncogenes (21-23). PSD4-1:14 overlaps in an antisense orientation with paired box 8 (PAX8), which belongs to the $P A X$ gene family, which plays a critical role in the formation of tissues and organs during embryonic development (24). PAX8 specifically is considered to activate genes involved in the formation of the thyroid gland and kidney $(25,26) . P A X 8$ has been previously characterized as a potential oncogene whose expression has been positively correlated with various types of epithelial and ovarian cancer (27-29). In addition, its overexpression has been associated with high levels of p53 (30). In those previous studies, $P A X 8$ was identified as a biomarker that could be used to differentiate between different types of epithelial tumors (31). It is possible that PSD4-1:14 acts as a cis-regulator of $P A X 8$, resulting in increased transcription of $P A X 8$, although this may not be the case, and the position of $P A X 8$ in the genome may not be associated with its interacting genes. To the best of our knowledge, $P A X 8$ has not been extensively studied in HNSCC; however, its role in other types of epithelial cancer suggests that it is candidate oncogene involved in the pathogenesis and progression of HNSCC. The exact molecular nature of the association between PSD4-1:14 and PAX8 has not been addressed in the present study, and further characterization of this association is a potential avenue of future research.

The cause of alcohol-associated HNSCC has not been previously characterized. Based on the results of the present study, it could be proposed that NETO1-1 and PSD4-1 may be partially responsible for the pathogenesis of alcohol-associated HNSCC, which highlights the importance of lncRNAs in the molecular mechanisms underlying the pathogenesis of HNSCC. While further studies are required to understand the exact mechanisms by which these lncRNAs function, PSD4-1 and NETO1-1 may be considered promising potential biomarkers and therapeutic targets of HSNCC. Further studies on these IncRNAs could potentially lead to innovations in the prevention and treatment of alcohol-induced HNSCC.

\section{Acknowledgements}

The clinical results described in the present study are in whole or partly based on data generated by the TCGA Research Network (http://cancergenome.nih.gov).

\section{References}

1. Hashibe M, Brennan P, Benhamou S, Castellsague X, Chen C, Curado MP, Dal Maso L, Daudt AW, Fabianova E, Fernandez L, et al: Alcohol drinking in never users of tobacco, cigarette smoking in never drinkers and the risk of head and neck cancer: Pooled analysis in the International Head And Neck Cancer Epidemiology Consortium. J Natl Cancer Inst 99: 777-789, 2007.

2. Boffetta P and Hashibe M: Alcohol and cancer. Lancet Oncol 7: 149-156, 2006.

3. Homann N, Jousimies-Somer H, Jokelainen K, Heine R and Salaspuro M: High acetaldehyde levels in saliva after ethanol consumption: Methodological aspects and pathogenetic implications. Carcinogenesis 18: 1739-1743, 1997.

4. Rahimy E, Kuo SZ and Ongkeko WM: Evaluation of non-coding RNAs as potential targets in head and neck squamous cell carcinoma cancer stem cells. Curr Drug Targets 15: 1247-1260, 2014.

5. Deng G and Sui G: Noncoding RNA in oncogenesis: A new era of identifying key players. Int J Mol Sci 14: 18319-18349, 2013.

6. Reilly M: Role of non-coding RNAs in the neuroadaptation to alcoholism and fetal alcohol exposure. Front Genet 3: 70, 2012 
7. Laufer BI, Mantha K, Kleiber ML, Diehl EJ, Addison SM and Singh SM: Long-lasting alterations to DNA methylation and ncRNAs could underlie the effects of fetal alcohol exposure in mice. Dis Model Mech 6: 977-992, 2013.

8. Spizzo R, Almeida MI, Colombatti A and Calin GA: Long non-coding RNAs and cancer: A new frontier of translational research? Oncogene 31: 4577-4587, 2012.

9. Dinger ME, Amaral PP, Mercer TR, Pang KC, Bruce SJ, Gardiner BB, Askarian-Amiri $\mathrm{ME}, \mathrm{Ru} \mathrm{K}$, Soldà $\mathrm{G}$ Simons C, et al: Long noncoding RNAs in mouse embryonic stem cell pluripotency and differentiation. Genome Res 18: 1433-1445, 2008

10. Guttman M, Amit I, Garber M, French C, Lin MF, Feldser D, Huarte M, Zuk O, Carey BW, Cassady JP, et al: Chromatin signature reveals over a thousand highly conserved large non-coding RNAs in mammals. Nature 458: 223-227, 2009.

11. Saxena A and Carninci P: Long non-coding RNA modifies chromatin: Epigenetic silencing by long non-coding RNAs. BioEssays 33: 830-839, 2011.

12. Rinn JL and Chang HY: Genome regulation by long noncoding RNAs. Annu Rev Biochem 81: 145-166, 2012.

13. Volders PJ, Helsens K, Wang X, Menten B, Martens L, Gevaert K, Vandesompele $\mathrm{J}$ and Mestdagh P: LNCipedia: A database for annotated human lncRNA transcript sequences and structures. Nucleic Acids Res 41 (Database issue): D246-D251, 2013.

14. Quinlan AR and Hall IM: BEDTools: A flexible suite of utilities for comparing genomic features. Bioinformatics 26: 841-842, 2010.

15. Robinson MD, McCarthy DJ and Smyth GK: edgeR: A Bioconductor package for differential expression analysis of digital gene expression data. Bioinformatics 26: 139-140, 2010.

16. Homann N, Tillonen J, Meurman JH, Rintamäki H, Lindqvist C, Rautio M, Jousimies-Somer $\mathrm{H}$ and Salaspuro M: Increased salivary acetaldehyde levels in heavy drinkers and smokers: A microbiological approach to oral cavity cancer. Carcinogenesis 21 : 663-668, 2000.

17. Livak, KJ and Schmittgen TD: Analysis of relative gene expression data using real-time quantitative PCR and the $2-\Delta \Delta C T$ method. Methods $25.4: 402-408,2001$

18. Prensner JR, Iyer MK, Balbin OA, Dhanasekaran SM, Cao Q, Brenner JC, Laxman B, Asangani IA, Grasso CS, Kominsky HD, et al: Transcriptome sequencing across a prostate cancer cohort identifies PCAT-1, an unannotated lincRNA implicated in disease progression. Nat Biotechnol 29: 742-749, 2011.
19. Gupta RA, Shah N, Wang KC, Kim J, Horlings HM, Wong DJ, Tsai MC, Hung T, Argani P, Rinn JL, et al: Long non-coding RNA HOTAIR reprograms chromatin state to promote cancer metastasis. Nature 464: 1071-1076, 2010.

20. Yang QQ and Deng YF: Long non-coding RNAs as novel biomarkers and therapeutic targets in head and neck cancers. Int J Clin Exp Pathol 7: 1286-1292, 2014.

21. Morris KV: Long antisense non-coding RNAs function to direct epigenetic complexes that regulate transcription in human cells. Epigenetics 4: 296-301, 2009.

22. Carrieri C, Cimatti L, Biagioli M, Beugnet A, Zucchelli S, Fedele S, Pesce E, Ferrer I, Collavin L, Santoro C, et al: Long non-coding antisense RNA controls Uchl1 translation through an embedded SINEB2 repeat. Nature 491: 454-457, 2012.

23. Geisler S and Coller J: RNA in unexpected places: Long non-coding RNA functions in diverse cellular contexts. Nat Rev Mol Cell Biol 14: 699-712, 2013.

24. Kent WJ, Sugnet CW, Furey TS, Roskin KM, Pringle TH, Zahler AM and Haussler D: The human genome browser at UCSC. Genome Res 12: 996-1006, 2002.

25. Pasca di Magliano M, Di Lauro R and Zannini M: Pax8 has a key role in thyroid cell differentiation. Proc Natl Acad Sci USA 97 13144-13149, 2000

26. Narlis M, Grote D, Gaitan Y, Boualia SK and Bouchard M: Pax2 and pax 8 regulate branching morphogenesis and nephron differentiation in the developing kidney. J Am Soc Nephrol 18: 1121-1129, 2007

27. Muratovska A, Zhou C, He S, Goodyer P and Eccles MR: Paired-Box genes are frequently expressed in cancer and often required for cancer cell survival. Oncogene 22: 7989-7997, 2003.

28. Laury AR, Perets R, Piao H, Krane JF, Barletta JA, French C, Chirieac LR, Lis R, Loda M, Hornick JL, et al: A comprehensive analysis of PAX8 expression in human epithelial tumors. Am J Surg Pathol 35: 816-826, 2011.

29. Liliac L, Carcangiu ML, Canevari S, Căruntu ID, Ciobanu Apostol DG, Danciu M, Onofriescu M and Amălinei C: The value of PAX8 and WT1 molecules in ovarian cancer diagnosis. Rom J Morphol Embryol 54: 17-27, 2013.

30. Brunner AH, Riss P, Heinze G, Meltzow E and Brustmann H: Immunoexpression of PAX 8 in endometrial cancer: Relation to high-grade carcinoma and p53. Int J Gynecol Pathol 30: 569-575, 2011.

31. Xiang L and Kong B: PAX8 is a novel marker for differentiating between various types of tumor, particularly ovarian epithelial carcinomas. Oncol Lett 5: 735-738, 2013. 